Article

\title{
Application of S-Transform Random Consistency in Inverse Synthetic Aperture Imaging Laser Radar Imaging
}

\author{
Bo Zang, Mingzhe Zhu *, Xianda Zhou, Lu Zhong® and Zijiao Tian \\ School of Electronic Engineering, Xidian University, Xi'an 710071, China; bzang@mail.xidian.edu.cn (B.Z.); \\ zhouxianda999@hotmail.com (X.Z.); zhonglu@protonmail.com (L.Z.); zijiao.tian@uconn.edu (Z.T.) \\ * Correspondence: zhumz@mail.xidian.edu.cn
}

Received: 8 May 2019; Accepted: 3 June 2019; Published: 5 June 2019

\begin{abstract}
Under the same principle, laser radar could be more sensitive to the micro-Doppler (m-D) effect due to its wave length, as the characteristic of multi-resolution, $\mathrm{S}$ transform could reduce the influence of the micro-Doppler component and enhance the imaging effect. This paper presents a method for micro-Doppler feature extraction in Inverse Synthetic Aperture Imaging Laser Radar (ISAIL) imaging. It is accessible and comprehensive, applying Random Sample Consensus (RANSAC) for the separation and reconstruction of micro-Doppler and rigid body signals. Experiments show that the method can effectively remove the micro-Doppler information and obtain a clear target distance-instantaneous Doppler image.
\end{abstract}

Keywords: time-frequency analysis; S-transformation; inverse synthetic aperture imaging laser radar; micro-Doppler; RANSAC

\section{Introduction}

Radar imaging is the reconstruction of a radar target from recorded data. The traditional imaging method is to perform spectrum analysis using a two-dimensional Fourier transform. The Fourier transform can show which frequency components are contained in the signal, but cannot show how the frequency changes with time. The literature [1,2] proposes the use of time-frequency analysis for radar moving target imaging. Each time-frequency tool has different effects on radar imaging.

Mechanical vibration or rotation of a target or structures on the target may induce additional frequency modulations on the radar echo signal which generate sidebands about the target's Doppler frequency, called the micro-Doppler effect. It is mainly caused by the rotation of propellers or rotor wings on a plane. Up until now, various research has been done on the m-D effect. But most of it has been limited to the microwave approach only. The time-frequency analysis method separates target from micro-Doppler information [3-5]. Time-frequency analysis can be used in feature extraction or parameter estimation of m-D components [6,7]. The Viterbi algorithm divides the micro-Doppler components $[8,9]$. The windowed time-frequency analysis and the Radon transform are used to isolate or suppress the micro-Doppler element [10].

The S-transform (ST), a well-known time-frequency analysis tool, has been deeply researched since it was proposed by Stockwell in 1996 [11] and has been used in many fields such as radar signal processing [12,13] and disposal of medical signals [14,15]. In some cases, the S-transform is also considered a special case of wavelet transform, while the usual wavelet transform does not have an inverse transform. The S-transform has three major advantages. First, the S-transform is a linear transform with no cross-term interference. Second, the S-transform has a unique inverse transform. Finally, the S-transform has multiple resolutions, better time resolution at high frequency 
and better frequency resolution at low frequency. In view of third merit, S-transform is especially suitable for analyzing radar echo signals for imaging. Its high frequency domain resolution in the low frequency region can achieve accurate imaging of the target rigid body component while the time domain resolution advantage of the high frequency region can effectively provide the target micro-Doppler information.

In recent years, the micro-Doppler effect on laser radar has gradually attracted attention in the field of radar. Inverse synthetic aperture imaging laser (ISAIL) [16] is such a coherent laser radar, the micron-size working wavelength makes a sensitive detection of the $\mathrm{m}$-D effect of target. The impact of the micro-Doppler effect on ISAIL imaging is mainly reflected in two aspects. First, the micro-Doppler effect will make the Doppler broadening of the corresponding distance unit echo and the resulting ISAIL image will be affected by the micro-Doppler component. Strip-shaped interference makes it difficult to understand the target image and extract the target features. Second, the micro-Doppler component in the echo contains a wealth of moving component motion feature information, which can provide more accurate target dynamic features. This provides a new way to analyze target characteristics. Therefore, how to separate the micro-Doppler component from the target echo and ensure the integrity of the two signals to meet the requirements of ISAIL imaging and target moving component motion feature analysis has become an important research topic.

In order to solve the above problems, this paper proposes an algorithm based on Random Sample Consensus [17-19] (RANSAC) to separate rigid body and micro-Doppler information. Clear radar imaging is obtained by using S-transformation. Based on the RANSAC algorithm, the characteristics of a specific mode component are accurately and quickly extracted in a complex multi-component system and the target rigid body part is separated from the micro-Doppler component, extracting the rigid body part and separating the target moving part. The target imaging is more evident. The feature extraction of the micro-Doppler information can obtain more accurate target dynamic characteristics.

The structure of this paper is as follows: an introduction to the ISAIL time-frequency model is in Section 2; a review of S-transform is presented in Section 3; a brief introduction to the algorithm is given in Section 4; the results of numerical simulations and real data tests are presented in Section 5; and concluding remarks are given in Section 6.

\section{The Principle of ISAIL Time-Frequency Imaging}

ISAIL imaging is the mapping of target echoes in the distance-Doppler plane. The model of the ISAIL m-D effect is described as Figure 1 [16]. Assuming the echo density function of the target is $\rho_{(x, y)}$, the radar echo signal can be expressed as:

$$
S(t)=\exp \left[-j 4 \pi f_{0} R(t) / c\right] \int_{-\infty}^{+\infty} \int_{-\infty}^{+\infty} \rho_{(x, y)} \exp \left[-j 2 \pi\left(x f_{x}-y f_{y}\right)\right] d x d y
$$

where $f_{x}=2 f_{0} \cos \theta(t) / c, f_{y}=2 f_{0} \sin \theta(t) / c$.

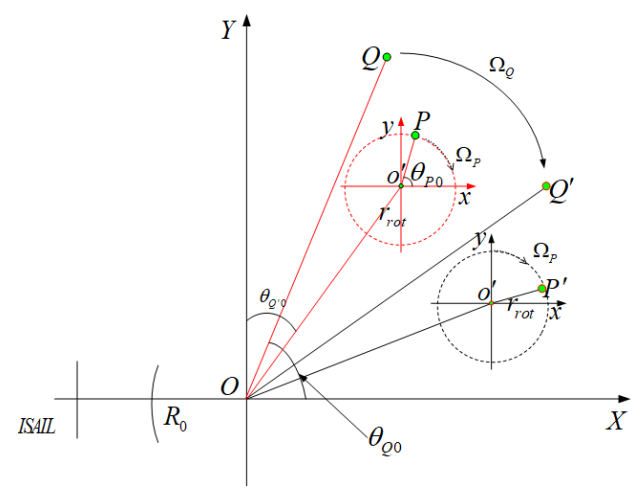

Figure 1. ISAIL geometric model. 
The distance $R(t)$ and rotation angle $\theta(t)$ of the instantaneous target can be determined by the initial position $R_{0}$ and $\theta_{0}$, the initial velocity $V_{0}$ and initial angular velocity $\omega_{0}$, the initial acceleration $a_{0}$ and initial angular acceleration $\alpha_{0}$ and other high-order terms.

$$
\begin{aligned}
& R(t)=R_{0}+v_{0} t+a_{0} t^{2} / 2 \\
& \theta(t)=\theta_{0}+\omega_{0} t+\alpha_{0} t^{2} / 2
\end{aligned}
$$

The purpose of ISAIL imaging is to find the scattering density distribution function from the radar echo. It can be seen from Equation (1) that the Doppler frequency can be decomposed into $f_{\text {trans }}$ and $f_{\text {rot }}$ :

$$
f_{D}=f_{\text {trans }}+f_{\text {rot }}=\frac{1}{2} \frac{d \phi(t)}{d t}=\frac{2 f_{0}}{c} v_{r}(t)+\frac{2 f_{0}}{c} \Omega(t) \times r_{c}(t)
$$

where $f_{\text {trans }}$ is generated by the target motion and it is determined by the radial velocity $v_{r}(t)$ of the target. $f_{\text {rot }}$ is generated by the rotation of the target and it is determined by the rotational speed $\Omega(t)$ of the target and the position $r_{c}(t)$ of the scattering point from the center of rotation. By motion compensation, $f_{\text {trans }}$ can be compensated for $f_{\text {rot }}$ to determine the position of the scattering point from the center of rotation. The traditional imaging method considers $f_{\text {rot }}$ to be constant, which can be established when the target is flying at low speed.

However, when the target is used for high-speed maneuvering, the Doppler spectrum is time-varying. If the conventional Fourier transform is used for spectrum analysis, the imaging will be blurred. In order to obtain high-resolution instantaneous Doppler spectra at various times, time-frequency analysis is required.

Figure 2 is the process of ISAIL time-frequency imaging. It is assumed that the accumulated pulse number is $\mathrm{M}$ and the number of distance units is $\mathrm{N}$ during the observation period. The time-frequency analysis is performed on the data of each distance unit. After the time sampling, the distance-Doppler ISAIL images of the $\mathrm{M}$ frame $N \times M$ can be obtained.

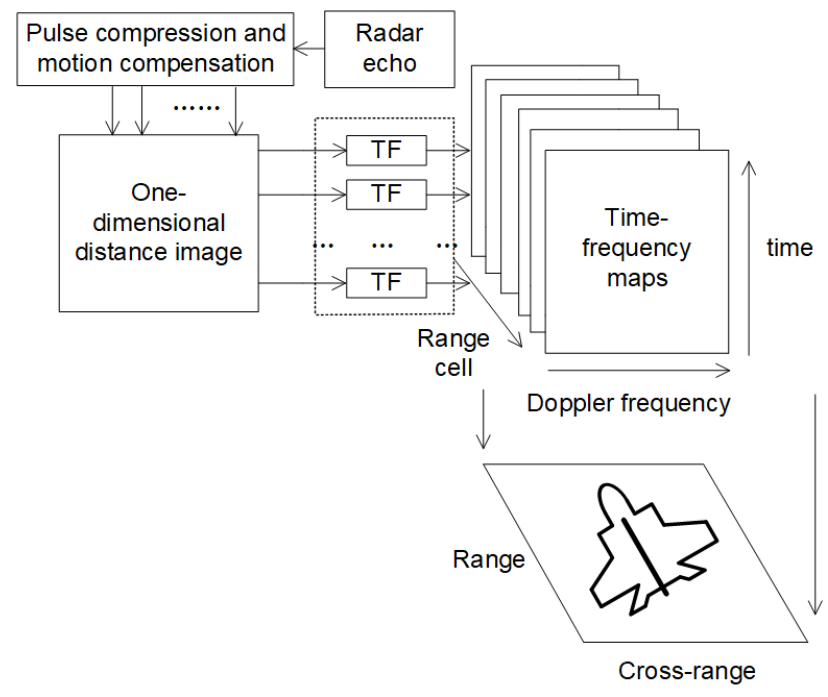

Figure 2. The process of ISAIL time-frequency imaging.

\section{S-Transform}

There are many methods for time-frequency analysis and the effects on ISAIL image imaging are also different. The Figure 3 shows the result of the short-time Fourier transform and S-transform for the same range cell. 


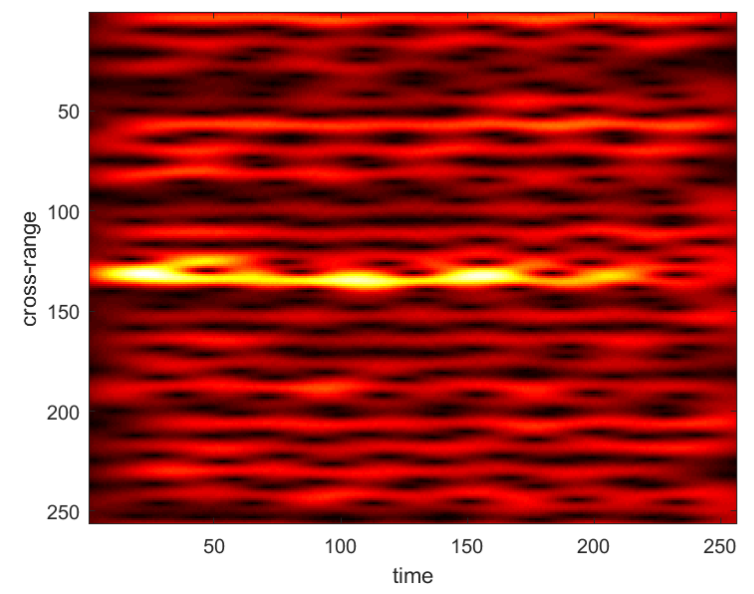

(a) STFT

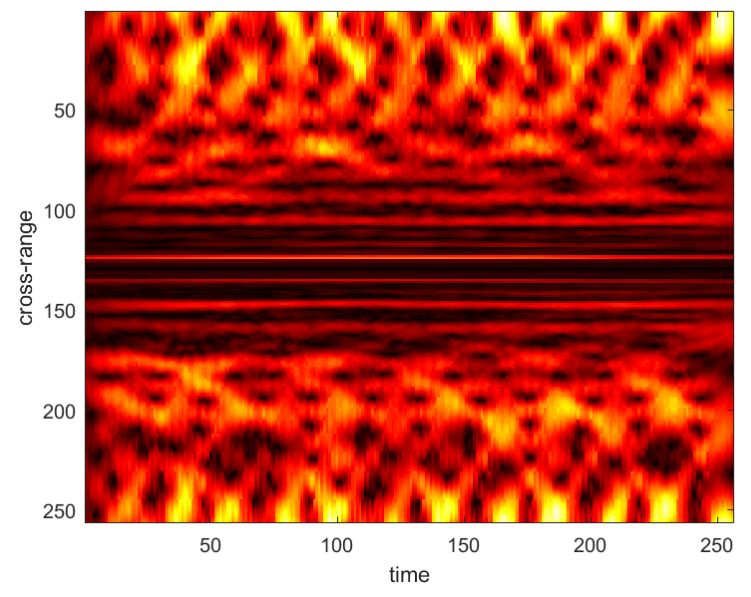

(b) ST

Figure 3. STFT and S-transform result of the same range cell.

The rigid body and micro-Doppler components of the radar can be approximated as single-frequency and sinusoidal modulated components. It can be seen from the figure that, due to the limitation of the window function, the distribution of the features of the rigid body and the micro-Doppler is not obvious in STFT. However, the S-transformation makes the target and micro-Doppler clearer, due to its enhanced diffusion into the high-frequency region.

The ST is proposed by American geophysicist Stockwell et al. in 1996. It is a method between the short-time Fourier transform and the wavelet transform. It uniquely combines the frequency dependence of time-frequency resolution and the localization of real and imaginary parts. The definition of the $\mathrm{S}$ transform:

$$
S_{x}(t, f)=\int_{-\infty}^{+\infty} x(\tau) \omega^{*}(\tau-t) e^{-j 2 \pi f \tau} d \tau
$$

$\omega(t)$ is a window function. According to the Heisenberg uncertainty principle, the time-frequency area of the Gaussian window function can be minimized. So the window function is selected as a Gaussian window:

$$
\begin{gathered}
\omega(t)=\frac{1}{\sigma \sqrt{2 \pi}} \exp \left[-\frac{t^{2}}{2 \sigma^{2}}\right] \\
\sigma(f)=\frac{1}{|f|}
\end{gathered}
$$

$\sigma$ is the scale factor that determines the width of the window function. It can be seen from the scale factor that at the high frequency , the signal changes drastically, the time period is relatively small, and a narrow time window is selected. While at the low-frequency, the signal change is relatively stable, the time period is relatively large and the time window is wider. At the same time, it is equivalent to adding a larger amplitude at the high frequency. It can be seen from Figure $4 a$ that the ST has a large amplitude at high frequencies (micro-Doppler information) and a small amplitude at low frequencies (rigid body portions), which is disadvantageous for the removal of the rigid body portion. Therefore, the data needs to be pre-processed first. This paper introduces the variable trend window $S$ transform [20].The scale factor in the window function has been improved.

$$
\sigma(f)=\frac{\frac{a}{3}[\lg 10(f+0.01)+1]}{|f|}
$$


Through the improved window function, the frequency resolution of the S-transform at high frequencies is improved and high-frequency distortion is suppressed. At the same time, the weight of the amplitude at high frequencies is reduced. The effectiveness of the micro-Doppler component is weakened.

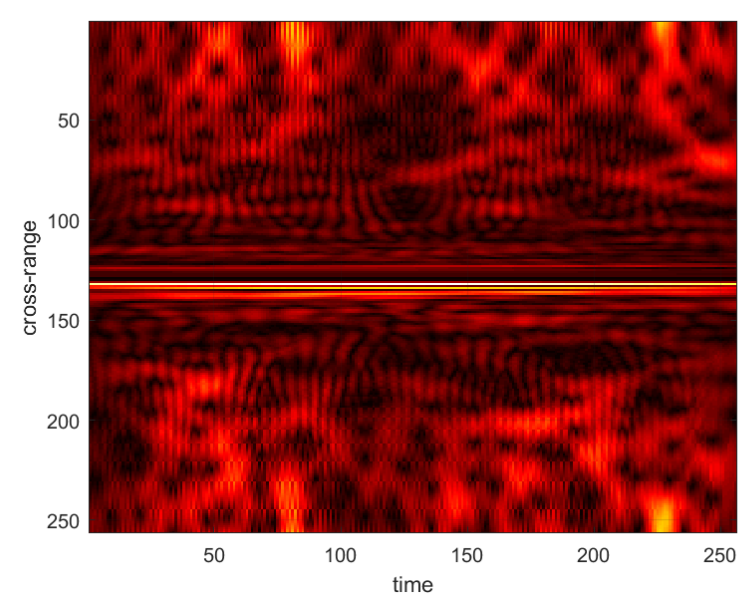

(a) ST result of 171th range cell

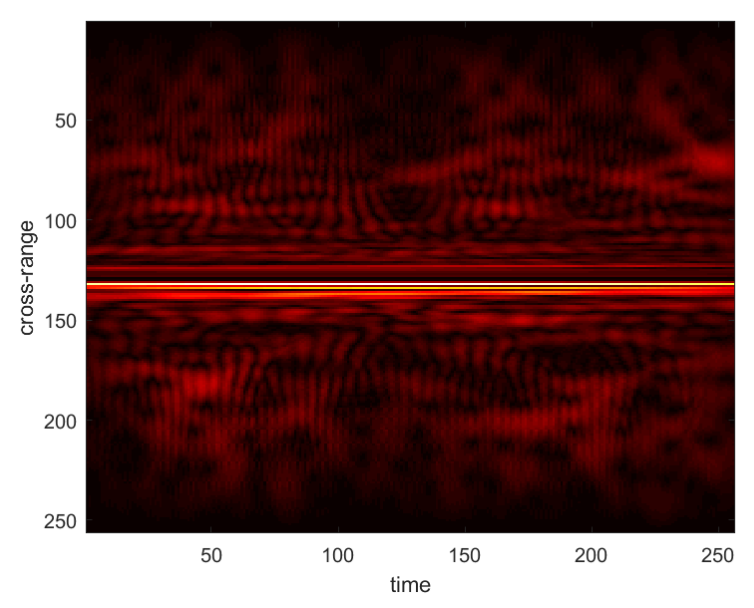

(b) Preprocessed ST result of 171th range cell

Figure 4. Preprocessed ST result.

After pre-processing, the amplitude of the high-frequency part becomes lower, highlighting the low-frequency part, which can better avoid the interference of the micro-Doppler information and more effectively take out the rigid body. Then the rigid body portion can be extracted by the RANSAC algorithm.

\section{Algorithm}

RANSAC is an algorithm that calculates the mathematical model parameters of the data based on a set of sample data sets containing abnormal data and obtains valid sample data. The rigid body and micro-Doppler portion of the radar can be approximated as single-frequency signals and sinusoidal modulated signals. A mathematical model of the single frequency signal can be established using the RANSAC algorithm to select a single frequency signal to remove abnormal data (sinusoidal modulated signal).

So the signal model can be modeled as:

$$
s(t)=\sum_{m=1}^{M} A_{m} \exp \left(j \phi_{m}(t)\right)
$$

$\phi_{m}(t)$ is the phase of the mth signal and $A_{m}$ is the amplitude..

$$
\phi_{m}(t)=\exp \left(j a_{m} \sin (2 \pi t)\right)+\exp \left(j b_{m} 2 \pi t\right)
$$

The RANSAC algorithm can select the components of the single-frequency signal to separate the rigid body and the micro-Doppler portion.

This algorithm's main steps are as follows:

Step 1: Calculate the time-frequency distribution of two dimensions (here, the ST).

Step 2: Pre-processing: penalize the weight of the S-transform at high frequencies, and perform the RANSAC algorithm. 
Step 3: Under a distance gate with micro-Doppler interference, the signal is divided into two parts. The target part is represented as a single-frequency signal and the micro-Doppler information part is represented as a sinusoidal modulated signal and the signal of the single-frequency signal is extracted by using the RANSAC algorithm.

Step 4: Repeat steps 3 to extract the rigid body parts of all the distance gates to obtain an ISAIL image without micro-Doppler interference.

\section{Simulation}

In order to verify the effectiveness of the proposed algorithm, it was tested with numerical simulation and real ISAIL m-D data. Simultaneously, compared with the use of singular-spectrum analysis extraction micro-Doppler features [16]. Simulation 1, the ISAIL data was simulated on the helicopter miniaturization model. The simulation parameters are shown in Table 1.

Table 1. Simulation parameters.

\begin{tabular}{cccc}
\hline Parameter/Variable & Value & Parameter/Variable & Value \\
\hline Center wavelength & $10.6 \times 10^{-6} \mathrm{~m}$ & Target size & $2.5 \mathrm{~m} \times 2.5 \mathrm{~m}$ \\
Transmitting bandwidth & $20 \times 10^{9} \mathrm{~Hz}$ & Velocity & $100 \mathrm{~m} / \mathrm{s}$ \\
Pulse repetition frequency & $7 \times 10^{3} \mathrm{~Hz}$ & Main rotor speed & $10 \mathrm{r} / \mathrm{s}$ \\
Coherent accumulation time & $0.1 \mathrm{~s}$ & Route angle relative to radar ray & $30^{\circ}$ \\
Transmitting width & $1 \mu \mathrm{s}$ & Imaging distance & $10 \mathrm{Km}$ \\
Range sampling points & 620 & Cross-range sampling points & 700 \\
\hline
\end{tabular}

The imaging results are shown in Figure 5. Figure 5a shows the helicopter model used in the simulation. Figure $5 \mathrm{~b}$ shows the result of distance pulse pressure. It is obvious that the micro-Doppler generated by the main rotor interferes with the echo signal of the helicopter body. Figure $5 \mathrm{c}$ is the results of imaging with S-transformation without separation of micro-Doppler information. Since the window function of the S-transform has amplitude weighting and the weighting value is 0 at zero frequency, there is a separate line at the zero frequency in the figure. However, it can still be seen that the helicopter body imaging results are significantly disturbed. Figure $5 \mathrm{~d}$ shows the result of imaging the helicopter main body after separating the micro-Doppler information by the singular-spectrum analysis [16] and Figure 5e is by the RANSAC method. It can be seen from the figure that the separated target features using the RANSAC algorithm are more obvious.

Simulation 2, the micro-Doppler feature extraction algorithm based on the RANSAC algorithm is verified by the real data of an Ann26 aircraft. The imaging results are shown in Figure 6. Figure 6a is an S-transform imaging result with significant micro-Doppler interference between the distance units 118-142. Figure $6 \mathrm{~b}$ shows the separated micro-Doppler component. In Figure $6 c$, the data processed by the singular-spectrum analysis [16] is shown and Figure $6 \mathrm{~d}$ is the data processed by the RANSAC algorithm. It all separates the rigid body portion from the micro-Doppler portion such as a propeller. However, it can be seen that Figure $6 \mathrm{c}$ lacks one wing of the aircraft and the features of Figure $6 \mathrm{~d}$ are more detailed. Therefore, the RANSAC algorithm is much better than the singular-spectrum analysis.

The above simulation experiments and the real data test show that the RANSAC based micro-Doppler feature extraction algorithm is effective for the processing of ISAIL target imaging and is much better than the singular-spectrum analysis. 


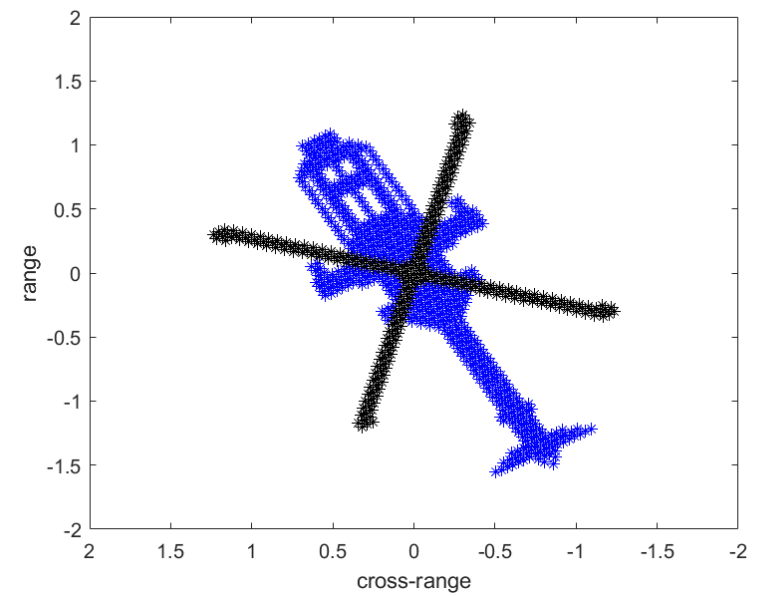

(a) The helicopter model

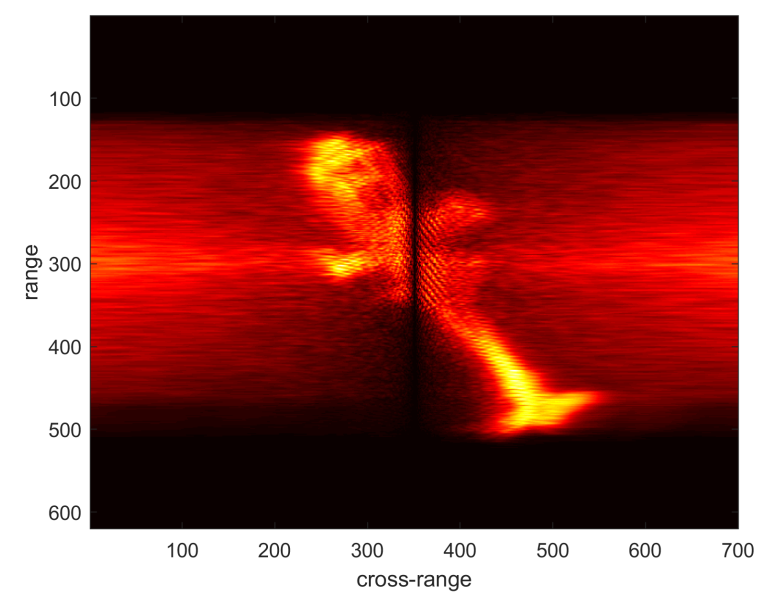

(c) The result of S-transformation imaging.

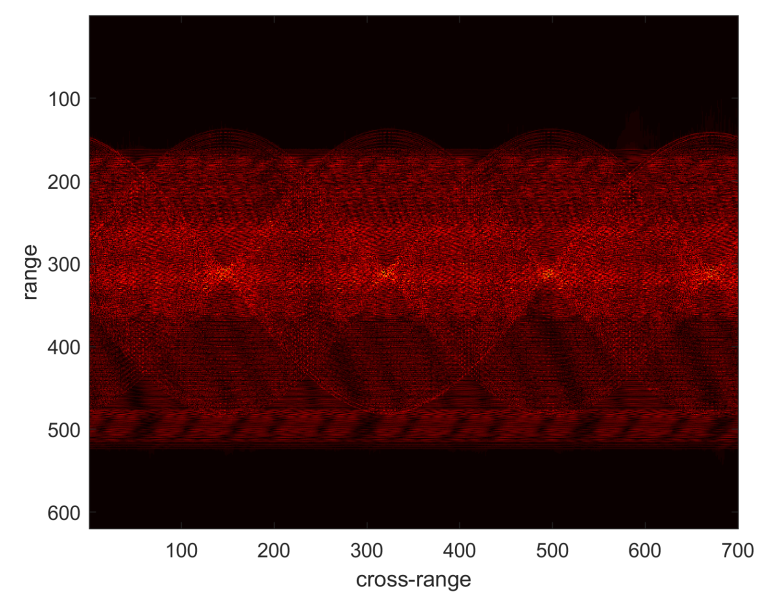

(b) The result of distance pulse pressure

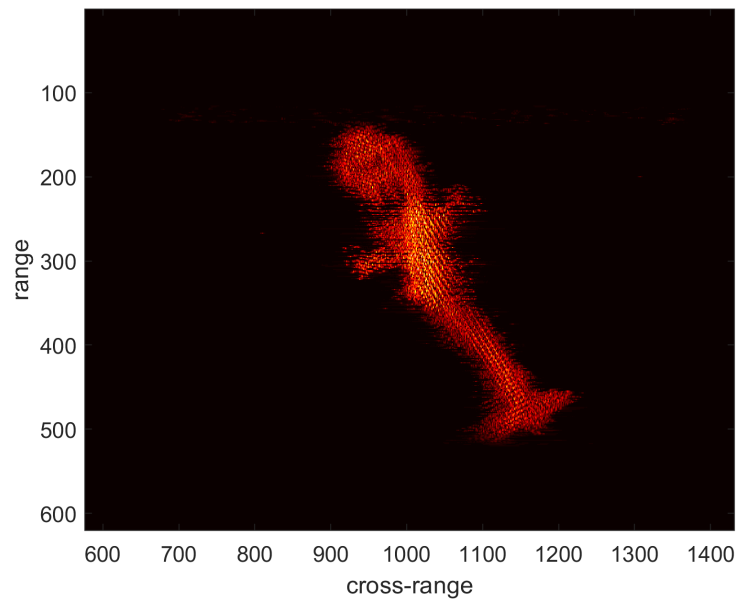

(d) The helicopter model separated by the singular-spectrum analysis

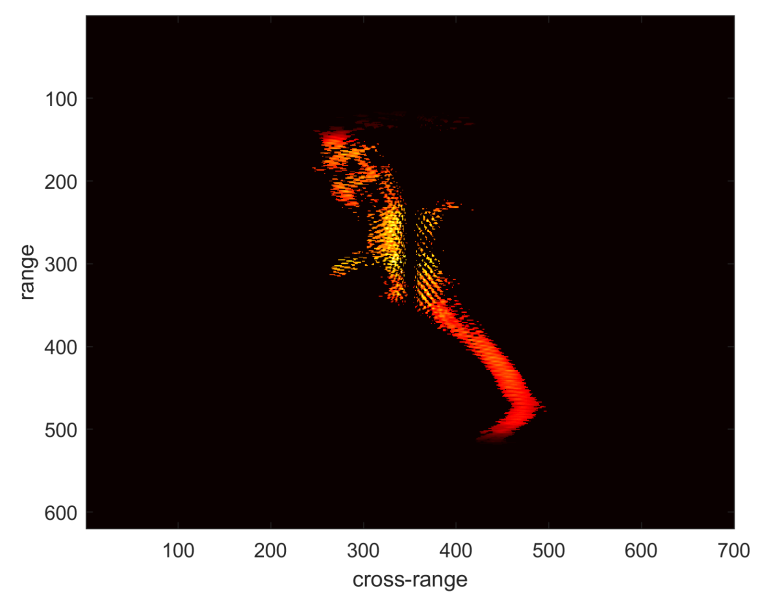

(e) The helicopter model separated by RANSAC

Figure 5. Simulation of the helicopter model. 


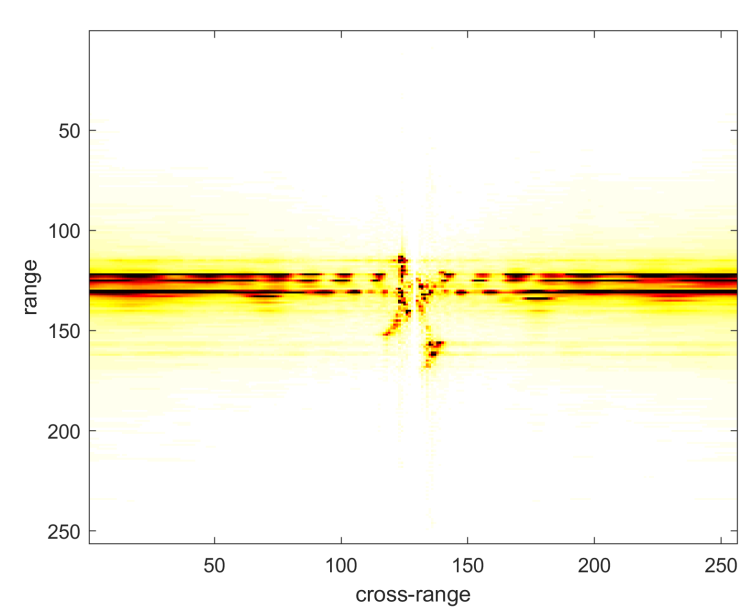

(a) S-transform imaging result.

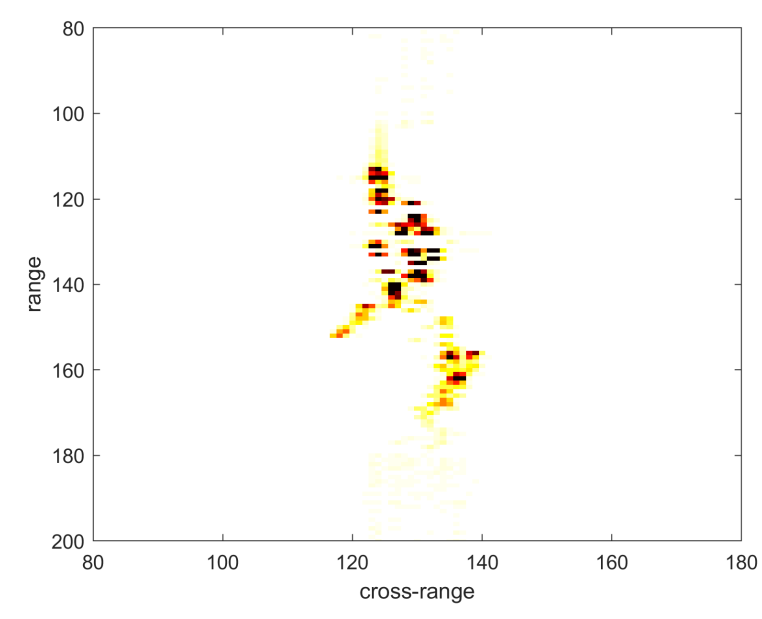

(c) The helicopter model separated by the singular-spectrum analysis

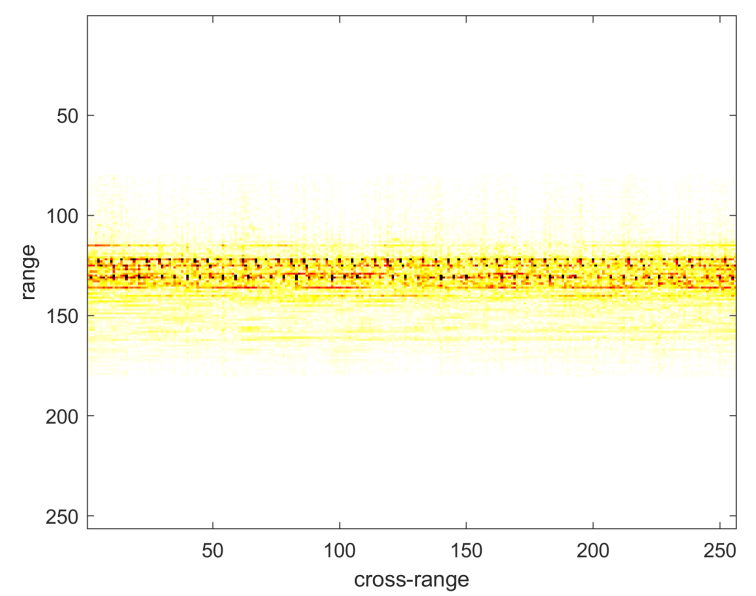

(b) The separated micro-Doppler component

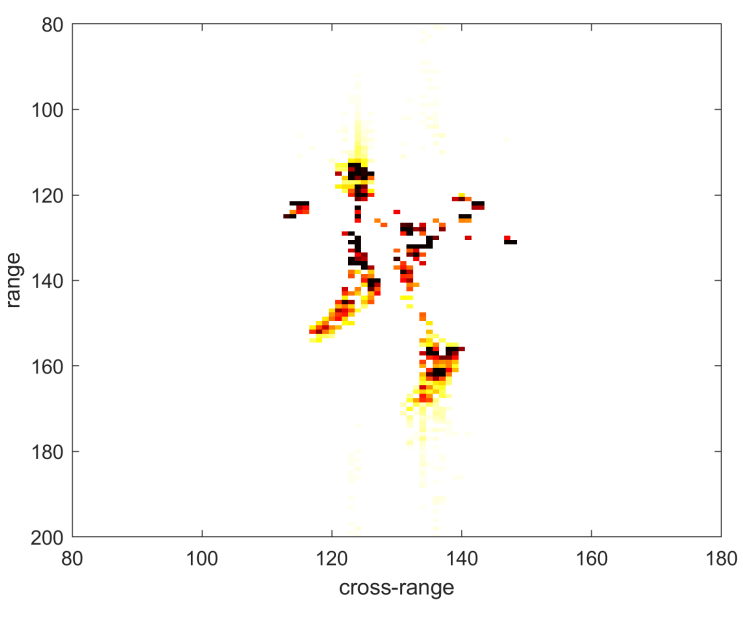

(d) The helicopter model separated by RANSAC

Figure 6. Imaging result of Ann26.

\section{Conclusions}

In this paper, a method based on S-transform and RANSAC for separating rigid body and micro-Doppler information is proposed. The RANSAC algorithm is used to separate the echo of the target rigid body part from the micro-Doppler information caused by the moving parts to obtain a clear ISAIL image. The simulation experiment and the measured data show that the proposed algorithm can extract the target rigid body part from the echo signal with micro-Doppler information and can obtain the target ISAIL image with high practical value. However, objective evaluation indicators for the results of various imaging and separation have not been given. The commonly used methods are not suitable for the data in this paper. In fact, the objective evaluation of images is a very complicated problem. Finding quantitative evaluation indicators that are consistent with subjective feelings will be a problem worthy of study in subsequent work. At the same time, the feature extraction of micro-Doppler information is under study.

Author Contributions: The contributions of the authors are as follows. Data curation, B.Z.; Formal analysis, M.Z.; Funding acquisition, X.Z.; Investigation, L.Z.; Methodology, Z.T.

Funding: This research was funded by National Natural Science Foundation of China grant number 61701374. 
Conflicts of Interest: The authors declare no conflict of interest.

\section{References}

1. Chen, V.C.; Ling, H. Time-Frequency Transforms for Radar Imaging and Signal Analysis; Artech House: Norwood, MA, USA, 2002.

2. Chen, V.C. Joint time-frequency analysis for radar signal and imaging. In Proceedings of the IEEE International Geoscience and Remote Sensing Symposium, Barcelona, Spain, 23-28 July 2007; pp. 5166-5169.

3. Chen, V.C.; Li, F.; Ho, S.S.; Wechsler, H. Analysis of micro-Doppler signatures. IEEE Proc.-Radar Sonar Navig. 2006, 42, 2-21. [CrossRef]

4. Chen, V.C.; Li, F.; Ho, S.S.; Wechsler, H. Micro-Doppler Effect in Radar: Phenomenon, Model, and Simulation Study. IEEE Proc.-Radar Sonar Navig. 2003, 150, 271-276. [CrossRef]

5. Li, J.; Ling, H. Application of adaptive chirplet representation for ISAR feature extraction from targets with rotating parts. IEEE Proc.-Radar Sonar Navig. 2003, 150, 284-291. [CrossRef]

6. Luo, Y.; Zhang, Q.; Qiu, C.; Li, S.; Yeo, T.S. Micro-Doppler feature extraction for wideband imaging radar based on complex image orthogonal matching pursuit decomposition. IET Radar Sonar Navig. 2013, 7, 914-924. [CrossRef]

7. Thayaparan, T.; Abrol, S.; Riseborough, E.; Stankovic, L.J.; Lamothe, D.; Duff, G. Analysis of radar micro-Doppler signatures from experimental helicopter and human data. IET Radar Sonar Navig. 2007, 1, 289-299. [CrossRef]

8. Li, P.; Wang, D.C.; Wang, L. Separation of micro-Doppler signals based on time frequency filter and Viterbi algorithm. Signal Image Video Process. 2013, 7, 593-605. [CrossRef]

9. Li, P.; Zhang, Q.H. An improved Viterbi algorithm for IF extraction of multicomponent signals. Signal Image Video Process. 2018, 12, 171-179. [CrossRef]

10. Stankovi'c, L.J.; Djurovi'c, I.; Thayaparan, T. Separation of target rigid body and micro-Doppler effects in ISAR imaging. IEEE Trans. Aerosp. Electron. Syst. 2006, 42, 1496-1506. [CrossRef]

11. Yin, B.; He, Y.; Li, B.; Zuo, L.; Yuan, L. An adaptive SVD method for solving the pass-region problem in S-transform time-frequency filters. Chin. J. Electron. 2015, 24, 115-123. [CrossRef]

12. Gao, F.; Xue, X.; Sun, J.; Wang, J.; Zhang, Y. A SAR image despeckling method based on two-dimensional S transform shrinkage. IEEE Trans. Geosci. Remote Sens. 2016, 54, 3025-3034. [CrossRef]

13. Gao, F.; Zhang, Y.; Wang, J.; Sun, J. Fast Algorithm for Inverse Two-Dimensional S Transform and Its Application in Time-Frequency Filtering for SAR Image Despeckling. Chin. J. Electron. 2016, 25, 100-105. [CrossRef]

14. Assous, S.; Humeau, A.; Tartas, M.; Abraham, P.; L'Huillier, J.P. S-transform applied to laser Doppler flowmetry reactive hyperemia signals. IEEE Trans. Biomed. Eng. 2006, 53, 1032-1037. [CrossRef] [PubMed]

15. Pinnegar, C.R.; Khosravani, H.; Federico, P. Time-frequency phase analysis of Ictal EEG recordings with the S-transform. IEEE Trans. Biomed. Eng. 2009, 56, 2583-2593. [CrossRef] [PubMed]

16. Zhu, M.; Zhou, X.; Zang, B.; Yang, B.; Xing, M. Micro-Doppler Feature Extraction of Inverse Synthetic Aperture Imaging Laser Radar Using Singular-Spectrum Analysis. Sensors 2018, 18, 3303. [CrossRef] [PubMed]

17. Djurovi'c, I. A WD-RANSAC Instantaneous Frequency Estimator. IEEE Signal Process. Lett. 2016, $23,757-761$. [CrossRef]

18. Djurovi'c, I. QML-RANSAC: PPS and FM signals estimation in heavy noise environments. Signal Process. 2015, 130, 142-151. [CrossRef]

19. Djurovi'c, I. QML-RANSAC Instantaneous Frequency Estimator for Overlapping Multicomponent Signals in the Time-Frequency Plane. IEEE Signal Process. Lett. 2018, 25, 447-451. [CrossRef]

20. Zhu, M.Z.; Ji, H.B.; Lin, L.; Wang, L. Instantaneous frequency estimation of multicomponent FM signals based on directional S tranform. J. Syst. Eng. Electron. 2013, 35, $29-33$.

(C) 2019 by the authors. Licensee MDPI, Basel, Switzerland. This article is an open access article distributed under the terms and conditions of the Creative Commons Attribution (CC BY) license (http:/ / creativecommons.org/licenses/by/4.0/). 